\title{
Editor's Message to Special Issue of Understanding, Technology, and Application of Interaction
}

\author{
KoJI TsukAdA ${ }^{1, a)}$
}

This special issue is organized in conjunction with the "Interaction 2016" (General Chair: Itaru Kuramoto, Program Chair: Koji Tsukada) which was held from March 2nd to March 4th, 2016 at the Science Museum (for General Lectures and Interactive Presentations).

The symposium has been held every year since 1997 and has been providing places for various researchers to discuss latest technology \& information related to Human Computer Interaction. The research topics includes not only Computer Science (e.g., User Interface, CSCW, Visualization, I/O Device, Virtual/Mixed Reality, Ubiquitous Computing, and Software Engineering), but also Humanities (e.g., Cognitive Science, Social Science, Cultural Anthropology, Media Theory and Art). The symposium is sponsored by 5 special interest groups: human computer interaction (HCI), groupware and network services (GN), ubiquitous computing system (UBI), entertainment computing (EC), and digital contents creation (DCON). At Interaction 2016, 18 peer-reviewed general lectures and 214 interactive presentations were presented and the symposium gathered 754 participants.

Since research projects on HCI tend to make rapid progress, it is very important to provide opportunities to publish journal papers immediately. Therefore, we have planned the special issue of "Understanding, Technology, and Application of Interaction" in conjunction with Interaction symposium. This will be the 17th edition of the special issue since 1998.

As members of the editorial committee for this special issue, we selected 16 chief program committee members of the Interaction 2016, who worked as meta-reviewer of General Lectures. When the submitted papers were also presented at Interaction 2016, we arranged the same person as meta-reviewer for smooth \& deep understanding. As a judgment policy, we tried to select (possibly) valuable papers for readers, without caring about the acceptance rate. In result, 25 papers were submitted for this special issue, and the 15 papers were accepted (acceptance rate: $60 \%)$.

We hope this special issue will bring something of value to readers who are in the related fields of HCI. We also hope they become interested in the Interaction Symposium and submit papers or participate as audience.

For the editing of this special issue, I would like to thank all authors for their contributions. I also appreciate the voluntary work of all reviewers, as well as the remarkable efforts of the members of the Editorial Committee and Board listed below. Finally, I would like to express my profound gratitude for the Editorial Board and secretariats of JIP for all support.

\section{The Editorial Committee}

- Editor in-Chief:

Koji Tsukada (Future University Hakodate)

- Editorial Board:

Takashi Yoshino (Wakayama University)

- Editorial Committee:

Takayuki Ito (Ochanomizu University)

Yuichi Ito (Osaka University)

Asako Kimura (Ritsumeikan University)

Itaru Kuramoto (Kyoto Institute of Technology)

Yasuyuki Kono (Kwansei Gakuin University)

Masataka Goto (AIST)

Daisuke Sakamoto (The University of Tokyo)

Ichiro Siio (Ochanomizu University)

Buntarou Shizuki (University of Tsukuba)

Yasuyuki Sumi (Future University Hakodate)

Yoshinari Takekawa (Future University Hakodate)

Tsutomu Terada (Kobe University)

Masahiro Toyoura (Yamanashi University)

Hideyuki Nakanishi (Osaka University)

Yoshinori Hijikata (Osaka University)

Masaaki Fukumoto (Microsoft Research)

Toshiyuki Masui (Keio University)

\footnotetext{
Future University Hakodate
}

a) tsuka@mobiquitous.com 